DOI: $10.5216 /$ cab.v13i3.9927

\title{
FATORES DE RISCO RELACIONADOS À OCORRÊNCIA DO TIMPANISMO ESPUMOSO EM BOVINOS CRIADOS NA REGIÃO DO AGRESTE MERIDIONAL DO ESTADO DE PERNAMBUCO, BRASIL
}

\author{
Luiz Teles Coutinho ${ }^{1 *}$, José Augusto Bastos Afonso ${ }^{1}$, Nivaldo de AZEVÊdo Costa ${ }^{1}$, PierRe \\ CASTRo SOARES ${ }^{2}$, CARLa LoPES DE MENDONÇA ${ }^{1}$ \\ 1Médicos Veterinários da Clínica de Bovinos, Universidade Federal Rural de Pernambuco, Garanhuns, PE, Brasil. - \\ luiz.teles@oi.com.br e coutinholtc@gmail.com \\ 2Professor Doutor da Universidade Federal Rural de Pernambuco, Recife, PE, Brasil.
}

\section{RESUMO}

Com o objetivo de se analisar os principais fatores de riscos associados à ocorrência do timpanismo espumoso em bovinos, foi realizado um estudo retrospectivo de 60 casos clínicos da enfermidade, diagnosticados em bovinos atendidos na Clínica de Bovinos, Campus Garanhuns da Universidade Federal Rural de Pernambuco, no período entre janeiro de 1989 a dezembro de 2007. Analisaram-se 60 fichas clínicas das quais foram resgatadas informações como alimentação, sistema de criação, época do ano, período de lactação, sexo e idade dos animais. Constatouse que, dos 60 animais acometidos, 54 (90\%) recebiam dietas com alto teor de concentrados e 41 (68,33\%) possuíam a palma como um dos ingredientes alimentares. Para $48(80 \%)$ animais, o sistema de criação verificado era de semi-intensivo a intensivo. A maioria dos animais acometidos era fêmea (57/60 - 95\%); desses, 44 (84,08\%) encontravam-se em lactação. A maior ocorrência (62\%) dos casos de timpanismo espumoso foi registrada no período do verão. O fluido ruminal desses animais tinha o valor de $\mathrm{pH}$ que oscilava ente 7 e 8 , a consistência era espumosa e havia comprometimento da fauna. Conclui-se que a associação de fatores como a oferta de dietas ricas em concentrados, durante o período de verão, para vacas nos dois primeiros trimestres de lactação, criadas num sistema semi-intensivo a intensivo de produção, devem ser considerados na etiopatogenia do timpanismo espumoso em bovinos na região do Agreste Meridional do Estado de Pernambuco - Brasil.

PALAVRAS-CHAVE: epidemiologia; ruminantes; transtorno digestivo.

\section{RISK FACTORS RELATED TO TE OCCURRENCE OF FOAMY BLOAT IN BOVINE REARED IN SOUTHERN-CENTRAL SEMI-ARID REGION OF PERNAMBUCO STATE, BRAZIL}

\section{ABSTRACT}

With the aim of analyzing the effects of risk factors such as feed, raising system, season, lactation period, gender and animal age on the occurrence of foamy bloat in bovines, a retrospective study was carried out on 60 clinical cases of the illness in bovines treated at the Bovine Clinic of the Universidade Federal Rural de Pernambuco, Garanhuns Campus (Brazil), between January 1989 to December 2007. Epidemiologically, data on risk factors obtained from medical charts were related to the occurrence of foamy bloat. Among the 60 animals affected, $54(90 \%)$ received diets with a high content of concentrates; palm was one of the ingredients in the diet of 41 animals. The raising system was semi-intensive to intensive for $48(80 \%)$ animals. The majority of animals affected were females (57/60), 44 (84.08\%) of which were in the lactation phase. A greater occurrence of cases of foamy bloat $(62 \%)$ was recorded in summer. The ruminal fluid in these animals had a $\mathrm{pH}$ value that oscillated 
between 7 and 8, was of a foamy consistency and had a negative effect on the microbiota. The interrelation of risk factors such as diet rich in concentrate, intensive to semiintensive production system, summer (dry season), the first and second lactation period, associated to the

KEYWORDS: digestive disorder; epidemiology; ruminants.

\section{INTRODUÇÃO}

A bovinocultura de leite é uma atividade de grande importância econômica para o estado de Pernambuco, sobretudo para a mesorregião do agreste que, nesses últimos anos, tem assumido uma posição de destaque dentro de um cenário nacional extremamente favorável, no que diz respeito ao crescimento da produção e ao aumento das exportações. Sendo assim, por razões econômicas e para se atender essa demanda cada vez maior, tem-se exigido do setor intensas e constantes melhorias em seus sistemas de produção. Entretanto, esses avanços, que abrangem melhorias desde o padrão genético dos animais, qualidade das instalações, aperfeiçoamento nas práticas sanitárias e, principalmente, no modelo nutricional dos rebanhos, têm, paralelamente, contribuído para o surgimento de transtornos digestivos, com certa frequência e gravidade, os quais têm acarretado sérios prejuízos ao produtor e à atividade.

Dentre esses transtornos digestivos, destacase o timpanismo espumoso (TE), que ocorre nos bovinos sob duas distintas condições epidemiológicas. A primeira condição caracteriza-se pelo consumo de forragem constituída, principalmente, de trevo e alfafa, em que essas plantas são, primariamente, as responsáveis pelo desenvolvimento e formação da espuma (MÉNDEZ \& RIET-CORREA, 2000; DALTO et al., 2009). A segunda condição estaria associada à ingestão de dietas ricas em grãos e, sobretudo, de fina granulometria, por bovinos confinados (GLOCK \& DeGROOT, 1998). Independente da condição desencadeante do TE, a conduta terapêutica a ser adotada dependerá da intensidade do processo e da gravidade clínica do paciente, podendo ser conservadora ou cirúrgica (COUTINHO et al., 2009).

Relatos primários de casos de TE em bovinos, na região do Agreste de Pernambuco, têm sugerido que o surgimento desse distúrbio estava associado ao consumo de dietas ricas em grãos por bovinos submetidos a um sistema de produção semiintensivo a intensivo (AFONSO et al., 2001).

Em função da necessidade de maiores estudos no sentido de se compreender melhor os interaction of factors inherent to the individual animal and ruminal microbiota are important to the etiopathology of foamy bloat in the southern-central semi-arid region of the State of Pernambuco, Brazil.

principais fatores de riscos relacionados ao aparecimento do $\mathrm{TE}$ em bovinos na região do Agreste de Pernambuco e, para que se possa intervir na adoção de medidas preventivas, realizou-se estudo de alguns fatores epidemiológicos como a dieta, o tipo de sistema de criação, a raça, a idade dos animais, a época do ano e a fase da lactação, inerentes à ocorrência desse processo fermentativo em bovinos. Avaliaram-se, também, algumas características do fluido ruminal dos animais acometidos pelo TE.

\section{MATERIAL E MÉTODOS}

Foi realizado um estudo retrospectivo, no qual foram resgatadas informações referentes aos dados das fichas clínicas de 60 bovinos (sendo 57 fêmeas e três machos) acometidos por TE no período entre Janeiro de 1989 e Dezembro de 2007, que foram examinados e tratados na Clínica de Bovinos, Campus Garanhuns da Universidade Federal Rural de Pernambuco (UFRPE).

As informações analisadas nos registros das fichas clínicas desses animais foram relacionadas a fatores epidemiológicos como alimentação, tipo de sistema de criação, raça, idade dos animais, época do ano, fase da lactação e o período de evolução da doença. A análise das características do fluido ruminal (cor, odor, consistência, $\mathrm{pH}$ e viabilidade dos protozoários - \% de vivos, densidade e motilidade) foi realizada empregando-se o modelo indicado por DIRKSEN et al. (1993).

$\mathrm{Na}$ análise estatística, os dados foram tabulados e processados por meio do programa computacional Statistical Analysis System (SAS, 1985), realizando-se a análise de dispersão de frequências, considerando frequências absolutas e relativas dos fatores relacionados com a casuística da enfermidade, bem como análise de regressão dos casos de distúrbios digestivos com função temporal (SAMPAIO, 1998).

\section{RESULTADOS E DISCUSSÃO}

A dispersão pareada do total de casos de animais com distúrbios digestivos e aqueles com TE encontra-se na Figura 1 e a distribuição de dados 
absolutos do total de bovinos atendidos por ano, bem como aqueles que apresentaram distúrbios de ordem digestiva e, mais particularmente, os que foram diagnosticados com TE encontram-se na Tabela 1. Observou-se uma maior ocorrência (12 casos) do TE no ano de 1999, contrastando com a sua ocorrência nos demais anos, tal fato pode ser justificado pela existência de um intenso e prolongado período de seca (verão), haja vista a baixa precipitação pluviométrica registrada naquele ano (Tabela 2), o que contribui para maior escassez de forragem, obrigando os pecuaristas a alimentarem os animais com dietas ricas em grãos, além disso, a associação com outros fatores de risco pode ter contribuído para o surgimento de mais casos de TE nesse ano.

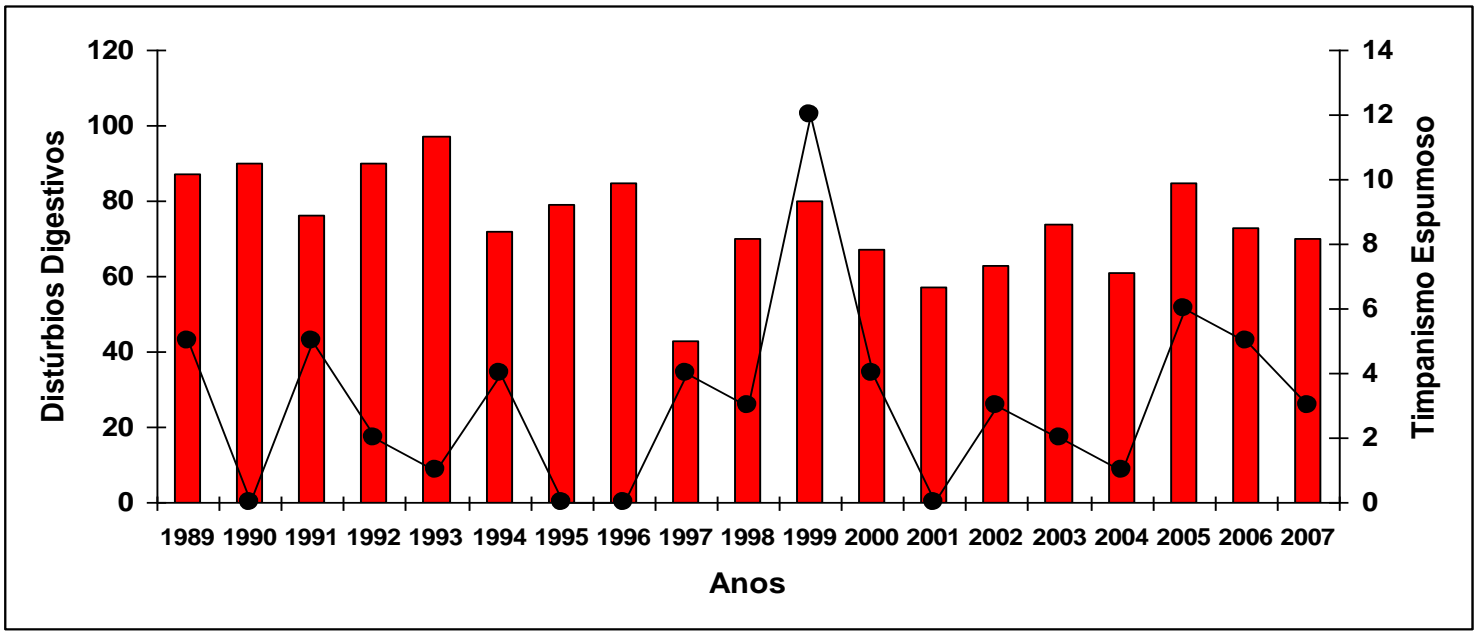

Figura 1. Número de casos de bovinos atendidos na Clínica de Bovinos - Campus Garanhuns - UFRPE com distúrbios digestivos e timpanismo espumoso no período de 1989 a 2007.

Tabela 1. Total de animais, casuística de distúrbios digestivos e timpanismo espumoso em bovinos atendidos na Clínica de Bovinos - Campus Garanhuns - UFRPE no período de 1989 a 2007

\begin{tabular}{ccccccc}
\hline \multicolumn{7}{c}{ Casuística } \\
\hline Anos & Bovinos & Digestivo & \% Dig/Total & T. Espumoso & \%TE/Total & \% TE/Dig \\
\hline 1989 & 540,00 & 87,00 & 16,11 & 5,00 & 0,93 & 5,75 \\
1990 & 509,00 & 90,00 & 17,68 & 0,00 & 0,00 & 0,00 \\
1991 & 393,00 & 76,00 & 19,34 & 5,00 & 1,27 & 6,58 \\
1992 & 438,00 & 90,00 & 20,55 & 2,00 & 0,46 & 2,22 \\
1993 & 411,00 & 97,00 & 23,60 & 1,00 & 0,24 & 1,03 \\
1994 & 482,00 & 72,00 & 14,94 & 4,00 & 0,83 & 5,56 \\
1995 & 454,00 & 79,00 & 17,40 & 0,00 & 0,00 & 0,00 \\
1996 & 370,00 & 85,00 & 22,97 & 0,00 & 0,00 & 0,00 \\
1997 & 319,00 & 43,00 & 13,48 & 4,00 & 1,25 & 9,30 \\
1998 & 336,00 & 70,00 & 20,83 & 3,00 & 0,89 & 4,29 \\
1999 & 344,00 & 80,00 & 23,26 & 12,00 & 3,49 & 15,00 \\
2000 & 378,00 & 67,00 & 17,72 & 4,00 & 1,06 & 5,97 \\
2001 & 331,00 & 57,00 & 17,22 & 0,00 & 0,00 & 0,00 \\
2002 & 463,00 & 63,00 & 13,61 & 3,00 & 0,65 & 4,76 \\
2003 & 423,00 & 74,00 & 17,49 & 2,00 & 0,47 & 2,70 \\
2004 & 421,00 & 61,00 & 14,49 & 1,00 & 0,24 & 1,64 \\
2005 & 452,00 & 85,00 & 18,81 & 6,00 & 1,33 & 7,06 \\
2006 & 415,00 & 73,00 & 17,59 & 5,00 & 1,20 & 6,85 \\
2007 & 381,00 & 70,00 & 18,37 & 3,00 & 0,79 & 4,29 \\
\hline Total & 7860,00 & 1419,00 & 18,05 & 60,00 & 0.76 & 4,23 \\
\hline
\end{tabular}


Tabela 2. Precipitação pluviométrica $(\mathrm{mm})$ total anual, médias mensais e do período de inverno e verão de Janeiro de 1989 a Dezembro de 2007 das estações experimentais 82890 Arcoverde-PE, 82892 Pesqueira-PE e 82893 Garanhuns-PE. (Fonte: INMET) - INMET: Instituto Nacional de Meteorologia

\begin{tabular}{ccccccccccccc}
\hline \multicolumn{1}{c}{ Arcoverde } & \multicolumn{1}{c}{ Garanhuns } & \multicolumn{4}{c}{ Pesqueira } \\
\hline \multicolumn{4}{c}{ Média/ } & \multicolumn{4}{c}{ Média/ } & \multicolumn{3}{c}{ Média/ } \\
Anos & Total & Mês & Inverno & Verão & Total & Mês & Inverno & Verão & Total & Mês & Inverno & Verão \\
\hline 1989 & 879,7 & 73,3 & 448,1 & 431,6 & 913,6 & 76,1 & 752,2 & 161,4 & 933,4 & 77,8 & 709,9 & 223,5 \\
1990 & 462,5 & 38,5 & 300,0 & 162,5 & 469,3 & 39,1 & 456 & 13,3 & 357,9 & 29,8 & 281,5 & 76,4 \\
1991 & 628,7 & 52,4 & 417,0 & 211,7 & 883 & 73,6 & 670,5 & 212,5 & 460,1 & 38,3 & 182,1 & 278 \\
1992 & 872,6 & 72,7 & 312,9 & 559,7 & 516,2 & 43,0 & 401 & 115,2 & 206,7 & 17,2 & 202,9 & 3,8 \\
1993 & 284,1 & 23,7 & 231,4 & 52,7 & 490,3 & 40,9 & 282,6 & 207,7 & 270,8 & 22,6 & 133,5 & 137,3 \\
1994 & 618,9 & 51,6 & 535,5 & 83,4 & 1024,4 & 85,4 & 893,4 & 131 & 609,4 & 50,8 & 325,9 & 283,5 \\
1995 & 551,1 & 45,9 & 328,8 & 222,3 & 829,3 & 69,1 & 644,7 & 184,6 & 684,6 & 57,1 & 286,2 & 398,4 \\
1996 & 618,6 & 51,6 & 551,9 & 66,7 & 871,1 & 72,6 & 749,3 & 121,8 & 618,2 & 51,5 & 438 & 180,2 \\
1997 & 640,8 & 53,4 & 398,2 & 242,6 & 846,3 & 70,5 & 565,7 & 280,6 & 703,6 & 58,6 & 303,9 & 399,7 \\
1998 & 299,6 & 25,0 & 233,5 & 66,1 & 453,2 & 37,8 & 413,5 & 39,7 & 182,9 & 15,2 & 161,6 & 21,3 \\
1999 & 373,4 & 31,1 & 184,7 & 188,7 & 488,3 & 40,7 & 326,3 & 162 & 458,5 & 38,2 & 142,4 & 316,1 \\
2000 & 850,2 & 70,9 & 468,7 & 381,5 & 1057,6 & 88,1 & 796,1 & 261,5 & 647,9 & 54,0 & 394,8 & 253,1 \\
2001 & 652,1 & 54,3 & 408,0 & 244,1 & 807,1 & 67,3 & 542,6 & 264,5 & 348,1 & 29,0 & 185 & 163,1 \\
2002 & 526,0 & 43,8 & 225,1 & 300,9 & 1190,9 & 99,2 & 676 & 514,9 & 529,3 & 44,1 & 253,1 & 276,2 \\
2003 & 503,5 & 42,0 & 358,6 & 144,9 & 651,9 & 54,3 & 464,5 & 187,4 & 243,6 & 20,3 & 101,3 & 142,3 \\
2004 & 980,8 & 81,7 & 372,8 & 608,0 & 1183,7 & 98,6 & 797,8 & 385,9 & 526 & 43,8 & 232,5 & 293,5 \\
2005 & 800,4 & 66,7 & 401,6 & 398,8 & 1223,9 & 102,0 & 878,3 & 345,6 & 787,9 & 65,7 & 312,7 & 475,2 \\
2006 & 527,7 & 44,0 & 409,3 & 118,4 & 949,5 & 79,1 & 746,4 & 203,1 & 546,9 & 45,6 & 362 & 184,9 \\
2007 & 889,1 & 74,1 & 483,8 & 405,3 & 1078,3 & 89,9 & 818,4 & 259,9 & 448,7 & 37,4 & 247,3 & 201,4 \\
\hline & 629,5 & & 372,1 & 257,4 & 838,31 & & 625,016 & 213,29 & 503,39 & & 276,66 & 226,73 \\
\hline
\end{tabular}

Observa-se, na Figura 2, a dispersão do número de casos de bovinos com timpanismo em função do tempo (anos), verificando-se por meio da análise de regressão uma dispersão de grande variabilidade, expressa pela equação de terceiro grau, ou seja, a condição do manejo alimentar é alterada, em função da precipitação pluviométrica, para um maior ou menor grau de confinamento e, consequentemente, maior oferta de rações concentradas.

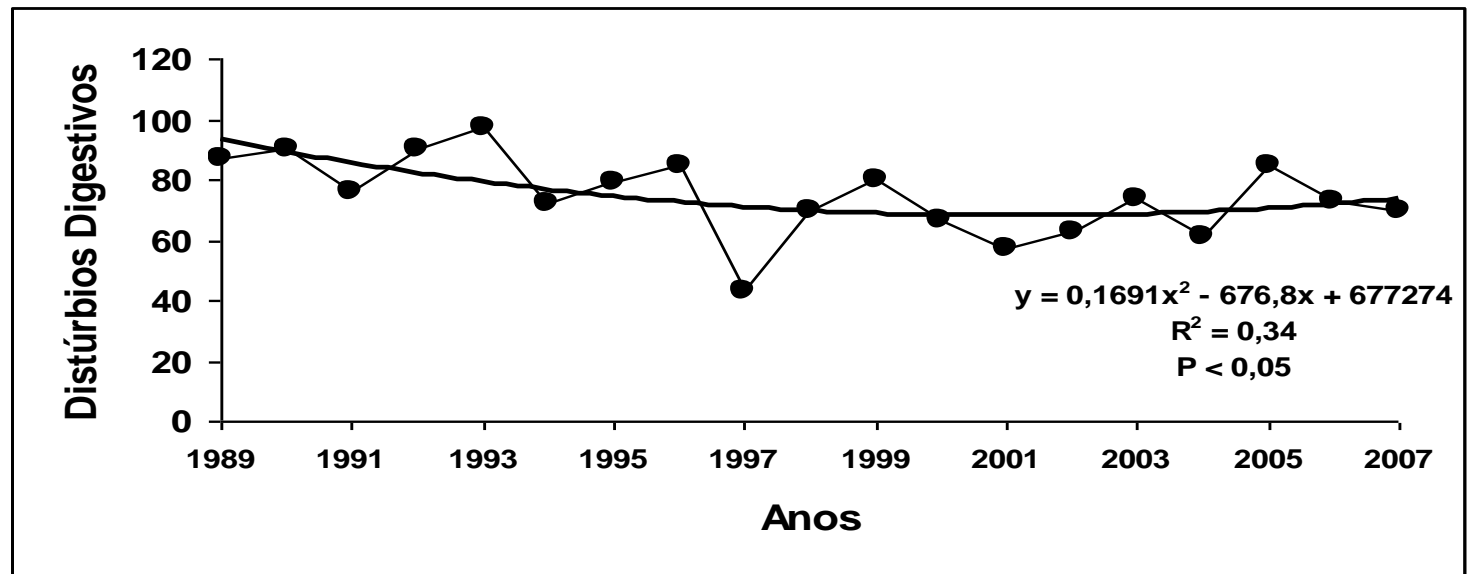

Figura 2. Representação gráfica da análise de regressão dos casos de distúrbios digestivos em função do tempo (anos) de bovinos atendidos na Clínica de Bovinos - Campus Garanhuns - UFRPE no período de 1989 a 2007. 
Nos animais acometidos, verificou-se que o período de evolução clínica da doença até o atendimento foi em torno de seis dias; entretanto, houve variação de animal para animal. Acreditase que a gravidade desse processo esteja relacionada com a composição da dieta e a interação com a população microbiana selecionada no ambiente ruminal (NAGARAJA et al., 1998).

Observou-se que a ocorrência do TE estava associada à ingestão de dietas com mais de $50 \%$ de concentrados (de fina ou baixa granulometria) em sua composição. Dos 60 animais acometidos por esse distúrbio, 54 (90\%) recebiam dietas com esse componente em sua formulação (Figura 3), fato corroborado por
COLE \& BODA (1960); CHENG et al. (1998); DIRKSEN et al. (2005), que relatam semelhante condição alimentar na ocorrência do TE, ou seja, animais que recebem dietas cuja composição tem mais de $50 \%$ de participação de concentrado. Aliado a isso, $41(68,3 \%)$ animais que tiveram TE recebiam dieta com palma, um alimento suculento, pobre em fibra, mas rico em mucilagem e carboidratos solúveis, que, em função da fermentação desses componentes, pode influir sobre a viscosidade do conteúdo ruminal, contribuindo para a não separação das bolhas de gás no interior do rumem e aumentando a estabilidade da espuma no processo inicial do desenvolvimento do TE (AMORIM et al., 2008).

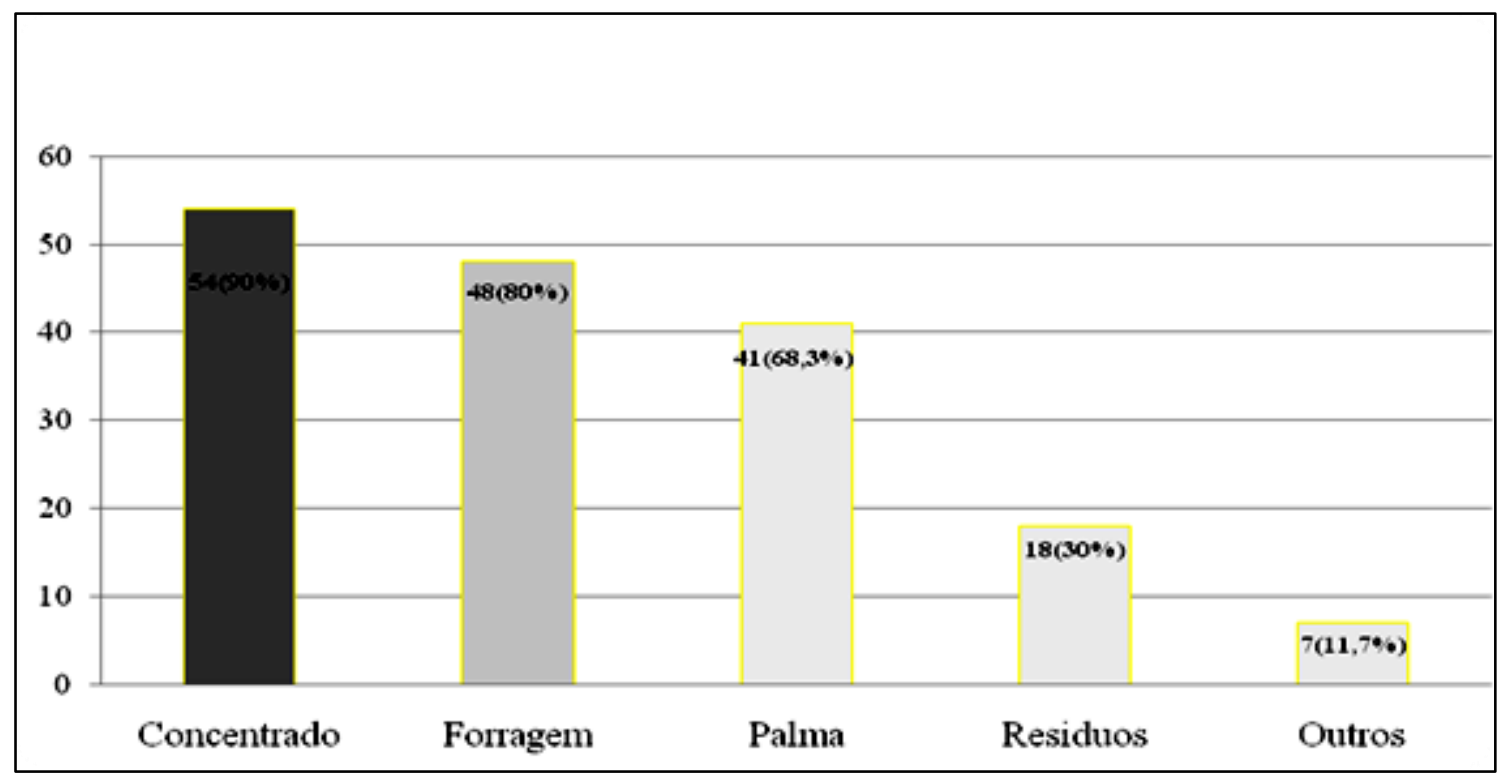

Figura 3. Tipos de componentes das dietas presentes nos casos de TE em bovinos. Análise de 60 casos.

Neste estudo, 48 (80\%) dos bovinos acometidos com TE estavam submetidos a sistema de produção semi-intensivo a intensivo, prática corriqueira em nosso meio, em função da caracterização da região que é constituída de muitas, porém pequenas propriedades, conforme constatado por MONTEIRO et al. (2007).
CLARKE \& REID (1974), COLVIN \& BACKUS (1988), GARRY (1990) e CHURCH (1993) afirmam que esse tipo de distúrbio fermentativo é frequente em bovinos e ovinos, principalmente com incidência elevada em propriedades onde se adota o modelo intensivo de produção (Figura 4). 


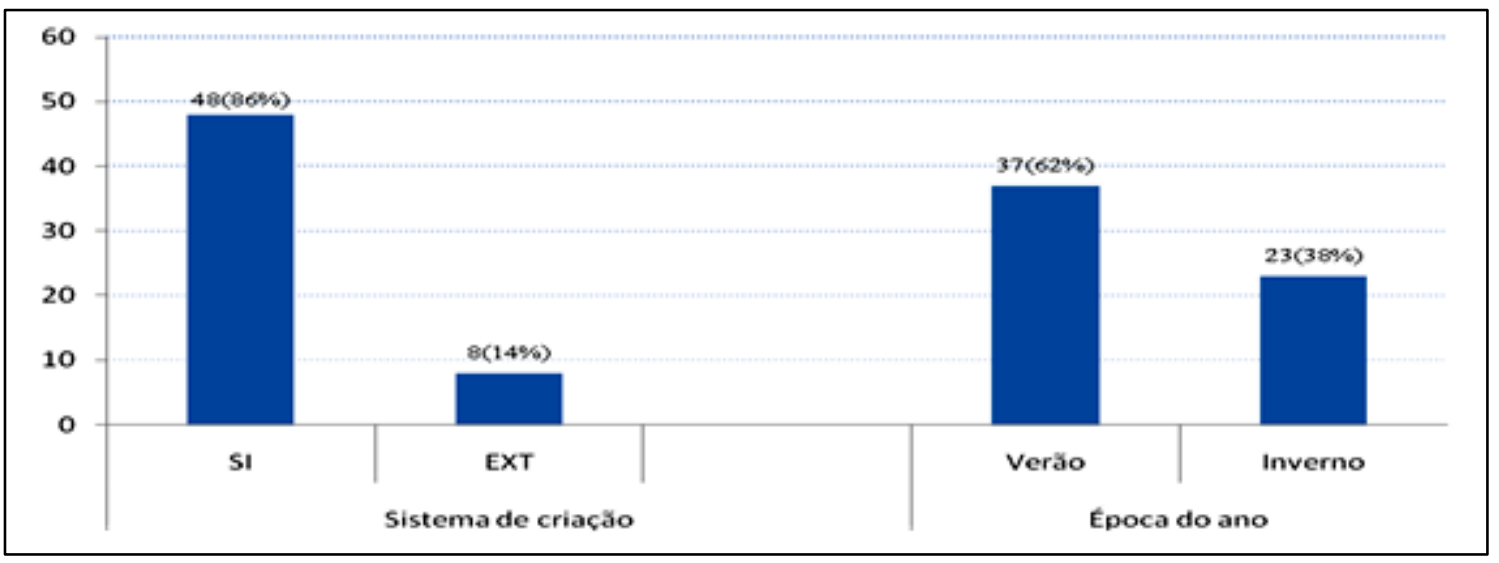

Figura 4. Tipo do sistema de criação e época do ano na ocorrência do TE em bovinos. Análise de 60 casos.

O sistema intensivo ou semi-intensivo de produção é adotado com o objetivo de viabilizar economicamente a propriedade; aliado às características produtivas e aos padrões raciais dos animais explorados na região, que caracteristicamente têm afinidade leiteira, aspecto facilmente observado neste trabalho, pois se verificou maior ocorrência da enfermidade nas fêmeas (apenas 3\% dos animais estudados eram machos). Considerando que as características da região do agreste meridional de Pernambuco são típicas de exploração leiteira, apresentando maior número de animais com essa aptidão, foi constatado no estudo que $75 \%$ dos animais com TE se enquadravam no padrão racial dos zebuínos, divergindo do padrão racial de animais de aptidão leiteira, que caracteristicamente é taurino. Entretanto, com esse fato não se pode afirmar que os zebuínos têm maior susceptibilidade ao TE que os taurinos, pois o cruzamento entre esses padrões raciais (Gir x Holandes) tem como produto o Girolando, que foi enquadrada na categoria zebuína, quando da tabulação dos dados, mas sabidamente destina-se à produção leiteira conforme estabelece ASSIS et al. (2005).

Ficou bem caracterizada, neste trabalho, a sazonalidade no surgimento do TE, tendo expressiva frequência no período de verão, quando $62 \%$ dos casos foram registrados (Figura 4). Assim como em todo o Nordeste, na região do Agreste Meridional de Pernambuco a existência de duas estações climáticas é bem definida: o inverno, época das chuvas, e o verão, época da seca, que correspondem aos períodos de abril a setembro e de outubro a março, respectivamente.

No verão, quando a baixa precipitação pluviométrica (Tabela 2) contribui para uma maior escassez de forragem, sobretudo de boa qualidade, faz-se necessário alimentar os animais com dietas à base de concentrados e de palma (buscando preservar a produtividade e até mesmo a mantença dos animais). Sendo assim, a exposição dos animais a esses fatores de risco que, em associação com algumas características da dieta, tais como sua forma física e tipo de grãos (granulometria da ração), e a interação com fatores inerentes ao individuo e aos microrganismos do rumem, contribuem positivamente para o aparecimento do TE conforme relatado por NAGARAJA (1998).

De 44 fêmeas registradas em fase produtiva, 37 (84,09\%) encontravam-se em lactação; dessas, 16 $(43,24 \%)$ estavam no primeiro trimestre da lactação, $15(40,54 \%)$, no segundo e seis $(16,22 \%)$, no terceiro. Já no período seco havia sete $(15,91 \%)$ animais (Figura 5). Tais achados sugerem que muitos desses animais poderiam se encontrar numa condição de balanço energético negativo (BEN) e, no intuito de se compensar os efeitos de tal transtorno, ofertavam-se dietas com elevadas quantidades de concentrado, muitas vezes de forma excessiva e sem a orientação de um plano nutricional adequado. A interação dessa prática com os fatores epidemiológicos já mencionados anteriormente cada vez mais potencializa o risco de surgimento do TE que, em nossa região, em função do seu impacto econômico, pode ser incluído na categoria dos transtornos digestivos de maior importância (VASCONCELOS \& GALYEAN, 2008). 


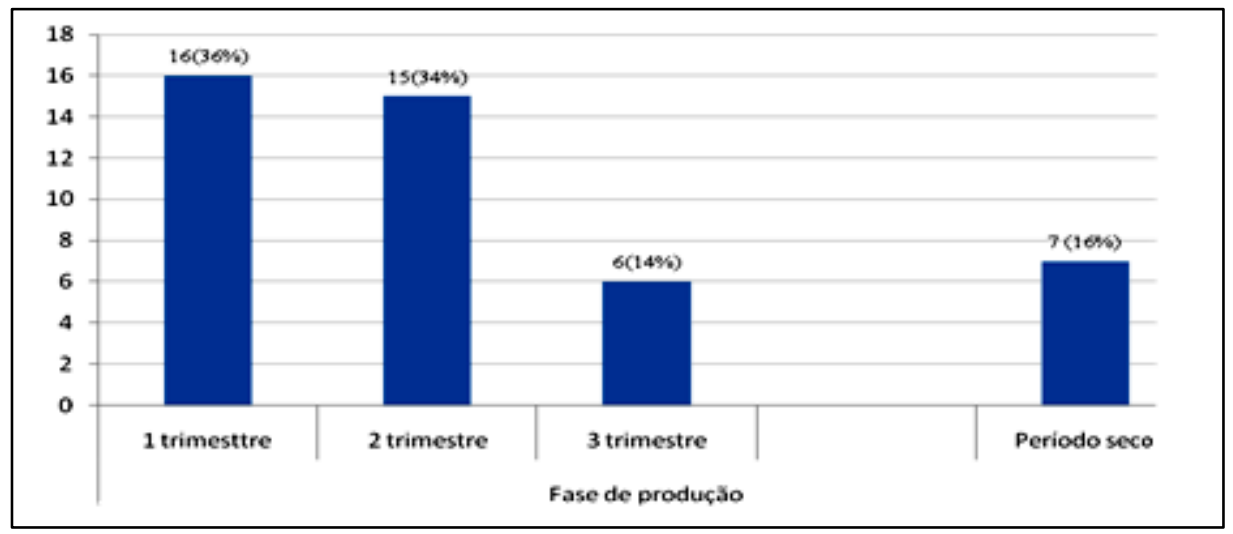

Figura 5. Fases de produção na ocorrência do TE em bovinos. Análise de 60 casos.

No fluido ruminal dos animais acometidos, foram evidenciadas alterações na consistência, que se apresentou em mais de $90 \%$ das amostras com aspecto de viscoso a espumoso (Figura 6), no comprometimento da densidade e motilidade da fauna e no $\mathrm{pH}$, que oscilou em maior frequência $(>80 \%)$ entre os valores de 7,0 a 8,0. Entretanto, os valore do $\mathrm{pH}$ podem estar mascarados em função do período de evolução da doença (seis dias) observado neste trabalho, que foi acompanhado de diminuição, e até ausência total de ingestão alimentar, o que contribui para a elevação do valor dessa variável (DIRKSEN et al., 1993). Corroborando com esse achado, MAJAK et al. (2003) afirmam que uma das condições determinantes para o estabelecimento de maior viscosidade do fluido ruminal é um $\mathrm{pH}$ relativamente baixo. No entanto, ELAM \& DAVIS (1962), CLARKE \& REID (1974), SAKAUCHI \& HOSHINO (1981) não associam a ocorrência do TE com valores específicos de $\mathrm{pH}$, exceto quando o mesmo ocorre paralelamente a um quadro de acidose ruminal. Já CAIRNIE (1981) relata que, para o TE causado por leguminosas, é necessário um pH de 5,8.

O comprometimento da fauna foi evidente na maioria das amostras ruminais examinadas; contudo, esse achado pode sofrer variação em diferentes proporções em bovinos com e sem a enfermidade (TE), conforme relata MISHRA (1969).

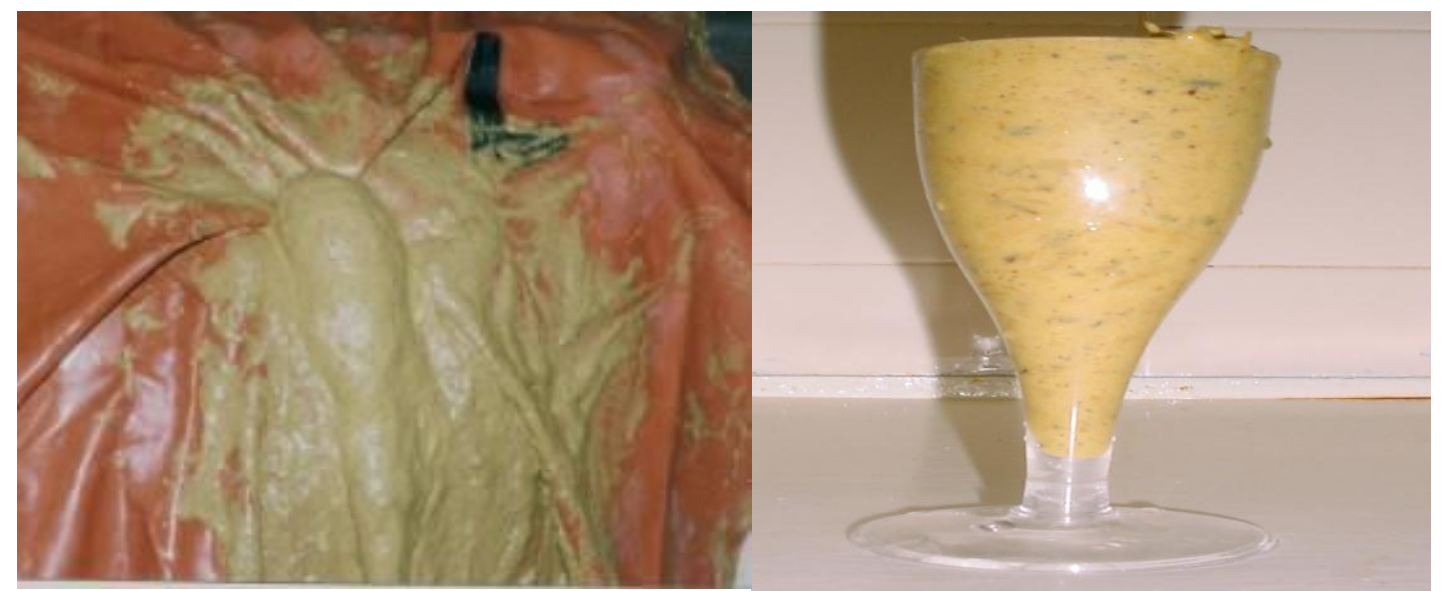

Figura 6. Fluido ruminal, de aspecto espumoso, obtido diretamente do rúmen, quando da ruminotomia.

\section{CONCLUSÃO}

Em função dos resultados e do que foi exposto, conclui-se que a associação dos fatores de risco estudados, como a oferta de dietas ricas em concentrados para vacas leiteiras nos dois primeiros trimestres de lactação, durante o período de verão, criadas semi e/ou intensivamente, confinadas na região do Agreste Meridional do Estado de Pernambuco - Brasil, deve ser considerada na 
etiopatogenia do timpanismo espumoso.

\section{REFERÊNCIAS}

AFONSO, J.A.B.; COSTA, N.A.; MENDONÇA, C.L.; SOUZA, M.I.; CALADO, A.C.; MIRANDA NETO, E.G.; LIMA, M.Z.P.R.; COUTINHO, L.T.; PIRES JR., J.B.; SIMÂO, L.C.V.; CAVALCANTE, A.E.L. Estudo retrospectivo do timpanismo espumoso em bovinos no Estado de Pernambuco. Ciência Veterinária nos Trópicos, v.4, n.2 e 3, p.249-255, 2001.

AMORIM, G.L.; BATISTA, A.M.V.; CARVALHO, F.F.R.; GUIM, A.; CABRAL, A.M.D.; MORAES, A.C.A. Substituição do milho por casca de soja: consumo, rendimento e características de carcaça e rendimento da buchada de caprinos. Acta Scientiarum: Animal Science, v.30, n.1, p.41-49, 2008.

ASSIS, A.G.; STOCK, L.A.; CAMPOS, O.F.; GOMEES, A.T.; ZOCCAL, R.; SILVA, M.R. Sistemas de produção de leite no Brasil. Circular Técnico [da] Embrapa Gado de Leite. n.85, p.1-6, 2005.

CAIRNIE, A.G. El meteorismo espumoso de los ruminantes. Secretaria de Agricultura y Ganaderia de La Nacion. Publicação Técnica [do] Instituto Nacional de Tecnologia Agropecuária, Estacion Experimental Agropecuária Anguil. n.23, p.66-96, 1981.

CHENG, K.J.; McALLISTER, T.A.; POPP, J.D.; HRISTOV, A.N.; MIR, Z.; SHIN, H.T. A review of bloat in feedlot cattle. Journal of Animal Science, v.76, n.1, p.299-308, 1998.

CHENG, K.J.; HIRONAKA, R.; JONES, G.A.; NICAS, T.; COSTERTON, J.W. Frothy feedlot bloat in cattle: production of extracellular polysaccharides and development of viscosity in cultures of Streptococcus bovis. Canadian Journal of Microbiology, v.22, n.4, p.450-459, 1976.

CHURCH, D.C. El Ruminante Fisiología Digestiva y Nutrición. Zaragoza: Acribia, 1993. 641p.

CLARKE, R.T.J.; REID, C.S.W. Foamy bloat of cattle. A review. Journal of Dairy Science, v.57, n.7, p.753-785, 1974.

COLE, H.H.; BODA, J.M. Continued progress towards controlling bloat. A review. Journal of Dairy Science. Champaign, n.43, p.1585-1614, 1960.

COLVIN, H.W.; BACKUS, R.C. Bloat in sheep. Compendium Biochemical Physiology, v.91, n.4, p.635644, 1988.

COUTINHO, L.T.; AFONSO, J.A.B.; COSTA, N.A; MENDONÇA, C.L.; FARIA, P.A.R.; SOARES, P.C. Avaliação da conduta terapêutica em casos de timpanismo espumoso em bovinos. Ciência Animal Brasileira, v.10, n.1, p.288-293, 2009.

DALTO, A.G.C.; BANDARRA, P.M., PEDROSO, P.M.O.; GUAGNINI, F.S.; LEAL, J.S.; RAYMUNDO,
D.L.; DRIEMEIER, D. Timpanismo espumoso em bovinos leiteiros em pastagens de Trifolium spp. (Leg. Caesalpinoideae). Pesquisa Veterinária Brasileira, v.29, n.5, p.401-403, 2009.

DIRKSEN, G.; GRÜNDER, H.D.; STÖBER, M. Medicina interna y cirugia del bovino. 4. ed. Buenos Aires: Inter-Médica, 2005. 406p. V.2.

DIRKSEN, G.; GRÜNDER, H.D.; STÖBER, M. Rosenberger exame clínico dos bovinos. 3. ed. Rio de Janeiro: Guanabara Koogan, 1993. 419p.

ELAM, C.J.; DAVIS, R.E. Ruminal characteristics and feedlot bloat incidence in cattle as influenced by vegetable oil, mineral oil, and animal fat. Journal of Animal Science, v.21, p.568-574, 1962.

GARRY, F.B. Managing bloat in cattle: symposium on bovine digestive disease. Veterinary Medicine, v.6, p.643-650, 1990.

GLOCK, R.D.; DEGROOT, B.D. Sudden death of feedlot cattle. Journal of Animal Science, v.76, p.315-319, 1998.

NSTITUTO NACIONAL DE METEOROLOGIA. Precipitação pluviométrica. Disponível em: <www.inmet.gov.br>. Acesso em: 29 set. 2009. (informação para assinante).

MAJAK, W.; McALLISTER, T.A.; McCARTNEY, D.; STANFORD, K.; CHENG, K.J. Bloat in cattle. [Edmonton]: Alberta Agriculture and Rural Development, 2003. 28p.

MÉNDEZ, M.C.; RIET-CORREA, F. Intoxicação por plantas e micotoxinas. In: __ Doenças dos Ruminantes e Eqüinos. São Paulo: Varela, 2000. p.219299.

MISHRA, B. Role of protozoa in rumen metabolism with special reference to bloat. Indian Veterinary Journal, v.46, n.1, p.42-49, 1969.

MONTEIRO, A.A.; TAMANINI, R.; SILVA, L.C.C.; MATTOS, M.R.; MAGNANI, D.F.; D'OVIDIO, L.; NERO, L.A.; BARROS, M.A.F.; PIRES, E.M.F.; PAQUEREAU, B.P.D.; BELOTI, V. Características da produção leiteira da região do agreste do estado de Pernambuco. Semina: Ciências Agrárias, v.28, n.4, p.665674, out.-dez. 2007.

NAGARAJA, T.G.; GALYEAN, M.L.; COLE, N.A. Nutrition and disease. In: STOKKA, G.L. Feedlot medicine and management. Veterinary Clinics of North America: Food Animal Practice, v.14, n.2, p.257-277, 1998.

SAKAUCHI, R.; HOSHINO, S. Microbial characteristics of ruminal fluid from feedlot bloat beef cattle. Journal of General and Applied Microbiology, v.27, p.145-155, 1981.

SAMPAIO, I.B.M. Estatística aplicada à experimentação animal. Belo Horizonte: Fundação de Ensino em Medicina Veterinária e Zootecnia, 1998. 221p.

STATISTICAL ANALYSIS SYSTEMS INSTITUTE. 
SAS User's Guide: Statistics. 5. ed. Cary, 1985. 912p. VASCONCELOS, J.T.; GALYEAN, M.L. ASAS Centennial Paper: Contributions in the Journal of Animal
Science to understanding cattle metabolic and digestive disorders. Journal of Animal Science, v.86: p.1711$1721,2008$.

Protocolado em: 16 maio 2010. Aceito em: 06 ago. 2012. 